\title{
Collision Avoidance of SDRE Controller using Artificial Potential Field Method: Application to Aerial Robotics*
}

\author{
S. R. Nekoo, J. Á. Acosta, and A. Ollero
}

\begin{abstract}
This work presents the problem of collision avoidance of the state-dependent Riccati equation (SDRE) controller using the artificial potential field (APF) method. Two themes were selected to illustrate the importance of the problem, collision avoidance between the end-effectors of serial links manipulators and unmanned aerial vehicles (UAVs), working in a shared workspace. The structure of the SDRE has a good potential to accommodate APF formulation in the weighting matrix of states. The distance between the end-effectors or the center-of-mass (CoM) of UAVs is penalized to autonomously guide the robots in a collision-free trajectory while they are working in a common environment. If the robots get close to each other, the weighting matrix of states increases, which actuates the systems to escape from a possible collision. Several simulation studies were done to investigate the proposed controller and the effect of collision avoidance function. It was found that the higher power of the collision avoidance function handles the threat of the impact better. The distance between robots was considered as an index to assess the performance of the controller which showed successful results in the simulations.
\end{abstract}

\section{INTRODUCTION}

Employing several robots to do a particular task has been highlighted in the literature and led to investigations in cooperative control and manipulations. Cooperation in robotics is getting more frequent in industrial applications, manufacturing production lines, and laboratories. The obvious result of several working drones or manipulators with complex structures and links is to face possible collisions between the systems during operations. To avoid this potential threat, a study of collision avoidance is essential.

The state-dependent Riccati equation is an optimal control policy, possessing nonlinear structure, that provides a good systematic and fruitful control law. The SDRE was introduced in the 1960s and became popular in the 1990s [1]. The application of the SDRE was investigated in robotics. Innocenti et al. presented the manipulator control via the statedependent Riccati equation [2]. The state-dependent coefficient (SDC) parameterization, and the experiment were provided to illustrate the performance of the SDRE approach. Terashima et al. studied the SDRE control in the servo design framework for a two-degree-of-freedom (DoF) planar manipulator [3]. No need for linearization was reported as the advantage of that method, resulting in the easier tuning of weighting matrices. Flexible-link robots were also controlled successfully by the SDRE, which presented very complicated dynamics including large and small deformations $[4,5]$.

Collision avoidance has been an interesting topic for UAV control. Multiple cooperative UAVs were employed for

*Research funded by the European Commission H2020 Programme under HYFLIER project contract 779411, AERIAL-CORE project contract number 871479, and the Spanish Project ARM-EXTEND DPI2017-89790-R detection and searching for a region while they were constrained to maintain in a close range and avoid possible collisions [6]. An optimal/suboptimal trajectory search was used to find the best path. A modified Grossberg neural network algorithm was proposed to overcome the collision avoidance problem for the same case [7]. A model predictive control was responsible for tracking the trajectory if that was a feasible solution. Defining a penalty for collisions in the fitness function for UAV trajectory optimization was proposed for free path planning [8]. Fathian et al. presented robust threedimensional distributed formation guidance with collision avoidance and application to multirotor aerial vehicles [9]. The presented research used a path generator for the first loop and a tracker for the second one. The feasibility of the generated trajectory was also an issue. This current work proposes a dynamic collision avoidance approach to provide the collisionfree trajectory in one control loop.

The subject of collision avoidance, in this work, employs the artificial potential field method which has a history in the SDRE control though limited to obstacle avoidance. Villagra et al. presented a flatness-based vehicle steering guidance method with SDRE feedback gains regulated by a sensitivity approach [10]. The obstacle avoidance terms were set in the weighting matrix of states. The same approach was used for manipulators [11], and a cable-driven robot satisfactorily [12]. Spacecraft proximity operation with obstacle constraint was investigated, considering the concept of finite-time stabilization [13]. Mohammadi and Khaloozadeh presented optimal motion planning of unmanned ground vehicles using the SDRE controller in the presence of obstacles [14]. Alirezaei et al. [15] presented a collision-avoidance design using a state-dependent Riccati equation control with experimental robustness assessment. The composite structure of the SDRE, compensative integral mode and artificial potential field method were used to formulate nonlinear control of spacecraft formation flying with an anti-disturbance system and collision avoidance [16]. Moving obstacle avoidance was also studied for wheeled mobile manipulators [17].

The application of multi-robot systems is recently being more visible in the control field. A multi-robot platform highlights the cooperation and the subsequent challenges in the dynamic structure of the plant. Korayem and Nekoo studied a nonlinear sub-optimal SDRE approach for cooperative manipulators to enhance dynamic load-carrying capacity (DLCC) [18]. Two manipulators carried an object in a predefined trajectory. Sharing the load between two arms was solved by an optimal load distribution method. Huang et al.

S. R. Nekoo, J. Á. Acosta, and A. Ollero are with GRVC Robotics Lab., Universidad de Sevilla, Seville, Spain; e-mails: saerafee@yahoo.com, \{jaar, aollero\}@us.es 
formulated an attitude take over control for the post-capture of target spaceship for a cooperative system [19].

This work incorporates the artificial potential field method within the SDRE control formalism to present collision avoidance safety guard for multi-robot systems, working in the shared environment. Setting the distance between the robots as denominators of weighting matrix $\mathbf{Q}(\mathbf{x})$ is the consequence of APF application. The subject of collision avoidance was once reported for wheeled mobile robots in Ref. [17], though the concept neither simulated nor analyzed for multiple manipulators or UAVs. So, the main contribution of this current research is to apply the concept of collision avoidance for a system of multiple manipulators and UAVs in a shared workspace. The system of robots, as a team, must avoid any collision in cooperation while they are close to other agents.

\section{The SDRE AND ARTIFICIAL Potential FiELD METHOD}

Consider a nonlinear system

$\dot{\mathbf{x}}(t)=\mathbf{f}(\mathbf{x}(t))+\mathbf{g}(\mathbf{x}(t), \mathbf{u}(t))$,

where $\mathbf{f}(\mathbf{x}(t)): \mathbb{R}^{n} \rightarrow \mathbb{R}^{n}$ and $\mathbf{g}(\mathbf{x}(t), \mathbf{u}(t)): \mathbb{R}^{n} \times \mathbb{R}^{m} \rightarrow \mathbb{R}^{n}$, are piecewise continuous nonlinear functions satisfying Lipschitz condition; $\mathbf{x}(t) \in \mathbb{R}^{n}$ and $\mathbf{u}(t) \in \mathbb{R}^{m}$, are state and input vector respectively. System (1) is transformed into the state-dependent coefficient parameterization [20]:

$\dot{\mathbf{x}}(t)=\mathbf{A}(\mathbf{x}(t)) \mathbf{x}(t)+\mathbf{B}(\mathbf{x}(t)) \mathbf{u}(t)$,

where $\mathbf{A}(\mathbf{x}(t)): \mathbb{R}^{n} \rightarrow \mathbb{R}^{n \times n}$ and $\mathbf{B}(\mathbf{x}(t)): \mathbb{R}^{n} \rightarrow \mathbb{R}^{n \times m}$ are held. The controllability condition: $\{\mathbf{A}(\mathbf{x}(t)), \mathbf{B}(\mathbf{x}(t))\}$, in (2), should present a completely controllable pair for all $\mathbf{x}$ and $t \in[0, \infty)$. The SDC parameterization must be designed in a way to guarantee the controllability of the $\{\mathbf{A}(\mathbf{x}(t)), \mathbf{B}(\mathbf{x}(t))\}$ in linear sense or at the equilibrium point $\{\mathbf{A}(\mathbf{0}), \mathbf{B}(\mathbf{0})\}$, considering that any term will not violate the controllability condition in the rest of the solution [21].

The performance index of SDRE possesses a quadratic form that tires leveling error rate and energy consumption [1]:

$J=\frac{1}{2} \int_{0}^{\infty}\left(\mathbf{x}^{T}(t) \mathbf{Q}(\mathbf{x}(t)) \mathbf{x}(t)+\mathbf{u}^{T}(t) \mathbf{R}(\mathbf{x}(t)) \mathbf{u}(t)\right) \mathrm{d} t$,

where $\mathbf{Q}(\mathbf{x}(t)): \mathbb{R}^{n} \rightarrow \mathbb{R}^{n \times n}$ and $\mathbf{R}(\mathbf{x}(t)): \mathbb{R}^{n} \rightarrow \mathbb{R}^{m \times m}$ are weighting matrices for states and inputs respectively. $\mathbf{Q}(\mathbf{x}(t))$ is symmetric positive semi-definite and $\mathbf{R}(\mathbf{x}(t))$ is symmetric positive definite.

In order to satisfy the observability condition, the pair of $\left\{\mathbf{A}(\mathbf{x}(t)), \mathbf{Q}^{1 / 2}(\mathbf{x}(t))\right\}$ must be a completely observable pair for all $\mathbf{x}$ and $t \in[0, \infty)$, in which $\mathbf{Q}^{1 / 2}(\mathbf{x}(t))$ is Cholesky decomposition of $\mathbf{Q}(\mathbf{x}(t))$. To guarantee the observability of the nonlinear pair $\left\{\mathbf{A}(\mathbf{x}(t)), \mathbf{Q}^{1 / 2}(\mathbf{x}(t))\right\}$ in (3), this pair must be observable in a linear sense similar to the controllability condition. The standard form of SDRE control law is found by applying stationary condition on Hamiltonian function as [5]:
$\mathbf{u}(t)=-\mathbf{R}^{-1}(\mathbf{x}(t)) \mathbf{B}^{T}(\mathbf{x}(t)) \mathbf{K}(\mathbf{x}(t)) \mathbf{x}(t)$,

where symmetric suboptimal control gain $\mathbf{K}(\mathbf{x}(t))$, is a solution to the state-dependent Riccati equation [22]:

$$
\begin{aligned}
& \mathbf{A}^{T}(\mathbf{x}) \mathbf{K}(\mathbf{x})+\mathbf{K}(\mathbf{x}) \mathbf{A}(\mathbf{x}) \\
& \quad-\mathbf{K}(\mathbf{x}) \mathbf{B}(\mathbf{x}) \mathbf{R}^{-1}(\mathbf{x}) \mathbf{B}^{T}(\mathbf{x}) \mathbf{K}(\mathbf{x})+\mathbf{Q}(\mathbf{x})=\mathbf{0} .
\end{aligned}
$$

The idea of obstacle avoidance is enlightened by an artificial potential field method [23]. To import the APF in the structure of the SDRE, $\mathbf{Q}(\mathbf{x}(t))$ in (5), the weighting matrix of states should be modified. It has the form of

$\mathbf{Q}(\mathbf{x}(t))=\left[\begin{array}{cccc}Q_{11}(\mathbf{x}(t)) & 0 & \cdots & 0 \\ 0 & Q_{22}(\mathbf{x}(t)) & \cdots & 0 \\ \vdots & \vdots & \ddots & \vdots \\ 0 & 0 & \cdots & Q_{n n}(\mathbf{x}(t))\end{array}\right]$,

where $Q_{i i}(\mathbf{x}(t))$ is $i$-th diagonal element of the matrix. If the value of any diagonal element in (6) increases, this results in a faster response of the relevant state. As a result, it is common to set the distance between the end-effector of a robot (or CoM of UAVs) and an obstacle as the denominator of $Q_{i i}(\mathbf{x}(t))$ to prevent any collision [11]. An SDRE regulator equipped with obstacle avoidance structure provides a controller with safe path planning capability. Collision avoidance benefits from the same point of view. If two or more states of two different systems should not collide with each other during a solution, the distance between them should be set as the denominator of diagonal elements of both or one of the systems $\mathbf{Q}_{1}(\mathbf{x}(t)), \mathbf{Q}_{2}(\mathbf{x}(t))$ :

$$
Q_{1, i i}(\mathbf{x}(t))=\frac{1}{\left(\sqrt{\sum_{j=1}^{m}\left[h_{1, j}(\mathbf{x}(t))-h_{2, j}(\mathbf{x}(t))\right]^{2}}\right)^{p}},
$$

where $p$ is a positive integer value, $h_{1, j}(\mathbf{x}(t))$ is $j$-th function of states for the first system and $h_{2, j}(\mathbf{x}(t))$ is $j$-th function for the second system; the value of $p>0$ defines the intensity of the collision avoidance. Here the system responsible for collision avoidance is system one, consequently $\mathbf{Q}_{1}(\mathbf{x}(t))$.

The collision avoidance term could be set in two systems though in some cases, this fails the generation of a safe path. Specifically, in a symmetric situation, two similar systems with symmetric initial and final conditions. In an event of a possible collision, both systems try to enhance the relative state to skip the collision. Nevertheless, they fail. This problem will be studied in the simulation section. So, it is strongly recommended to define one of the systems responsible for collision avoidance or set the collision avoidance term without any symmetric structure or gains.

Small or large workspace: The end-effectors of manipulators are usually working in a limited workspace, with the maximum distance between the end-effectors less than 1 meter. In that case $Q_{1, i i}(\mathbf{x}(t))$ in Eq. (7) increases as the collision distance decrease. Therefore, the responsible manipulator for collision avoidance tries to regulate faster towards the final condition. However, for a large workspace, control of UAVs in an open environment, the distance between 
the drones are usually more than 1 meter. In that case, $Q_{1, i i}(\mathbf{x}(t))$ acts oppositely and weakens the responsible UAV for obstacle avoidance in a possible collision. So, the following condition completes the collision avoidance strategy for the large workspace:

$\left\{\begin{array}{lll}\text { if } & d_{\mathrm{L}}<1 & Q_{1, i i}(\mathbf{x}(t))=\frac{1}{d_{\mathrm{L}}^{p}}, \\ \text { if } \quad d_{\mathrm{L}}>1 & Q_{1, i i}(\mathbf{x}(t))=d_{\mathrm{L}}^{p},\end{array}\right.$

where $d_{\mathrm{L}}$ is the distance function, representing the distance between the systems as

$$
d_{\mathrm{L}}=\sqrt{\sum_{j=1}^{m}\left[h_{1, j}(\mathbf{x}(t))-h_{2, j}(\mathbf{x}(t))\right]^{2}},
$$

in which $m$ is 2 for planer collision avoidance of end-effectors and 3 for 3D collision avoidance of UAVs.

\section{MOdELING OF A COOPERATIVE SYSTEM CONSIDERING \\ COLLISION AVOIDANCE}

\section{A. End-effector Collision Avoidance}

The case study of this section is dedicated to collision avoidance between the end-effectors of two manipulators, working in a shared workspace, Fig. 1. The left arm is placed at $(-d, 0)$ and the right arm at $(d, 0)$, with the assumption of symmetric structure and the same kinematics and dynamics parameters. The gravity, $g=9.81\left(\mathrm{~m} / \mathrm{s}^{2}\right)$, is present in $Y$ direction.

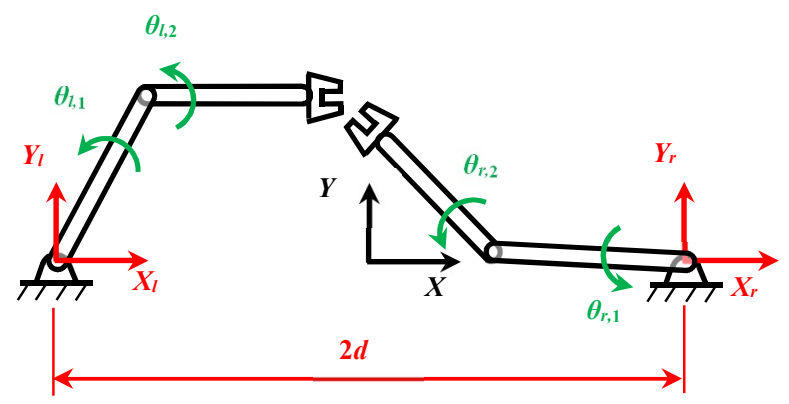

Figure 1. Two planar two-DoF manipulators, working in a shared workspace considering end-effector collision avoidance.

The generalized coordinates of the left arm are $\mathbf{q}_{l}=\left\{\theta_{l, 1}(t), \theta_{l, 2}(t)\right\}$ and the one for the right one is $\mathbf{q}_{r}=\left\{\theta_{r, 1}(t), \theta_{r, 2}(t)\right\}$. Consequently, using Lagrange method, the equation of motion of two systems are:

$$
\begin{aligned}
& \mathbf{M}_{l}\left(\mathbf{q}_{l}\right) \ddot{\mathbf{q}}_{l}+\mathbf{C}_{l}\left(\mathbf{q}_{l}, \dot{\mathbf{q}}_{l}\right) \dot{\mathbf{q}}_{l}+\mathbf{G}_{l}\left(\mathbf{q}_{l}\right) \mathbf{q}_{l}=\boldsymbol{\tau}_{l}, \\
& \mathbf{M}_{r}\left(\mathbf{q}_{r}\right) \ddot{\mathbf{q}}_{r}+\mathbf{C}_{r}\left(\mathbf{q}_{r}, \dot{\mathbf{q}}_{r}\right) \dot{\mathbf{q}}_{r}+\mathbf{G}_{r}\left(\mathbf{q}_{r}\right) \mathbf{q}_{r}=\boldsymbol{\tau}_{r},
\end{aligned}
$$

where $\left[\mathbf{M}_{i}\left(\mathbf{q}_{i}\right)\right]_{2 \times 2}$ is inertia matrix, $\left[\mathbf{C}_{i}\left(\mathbf{q}_{i}, \dot{\mathbf{q}}_{i}\right) \dot{\mathbf{q}}_{i}\right]_{2 \times 1}$ is Coriolis and centrifugal vector, $\left[\mathbf{G}_{i}\left(\mathbf{q}_{i}\right) \mathbf{q}_{i}\right]_{2 \times 1}$ is gravity vector and $\boldsymbol{\tau}_{i} \in \mathbb{R}^{2}$ is input torque vector; for $i=l, r$.

Consideration of gravity in SDC parameterization imposes a limitation in trajectory tracking design or regulation problem $\theta_{i, 1} \neq 0$; however, this design provides the optimal solution. The parameters of the robot and the definition of them could be followed in Ref. [24].

Considering the state vector of the entire cooperative system as $\quad \mathbf{x}(t)=\left[\mathbf{q}_{l}^{T}(t), \mathbf{q}_{r}^{T}(t), \dot{\mathbf{q}}_{l}^{T}(t), \dot{\mathbf{q}}_{r}^{T}(t)\right]^{T}$, the state-space representation is obtained:

$$
\dot{\mathbf{x}}(t)=\left[\begin{array}{c}
\dot{\mathbf{q}}_{l} \\
\dot{\mathbf{q}}_{r} \\
\mathbf{M}_{l}^{-1}\left(\mathbf{q}_{l}\right)\left[\boldsymbol{\tau}_{l}-\left(\mathbf{C}_{l}\left(\mathbf{q}_{l}, \dot{\mathbf{q}}_{l}\right) \dot{\mathbf{q}}_{l}+\mathbf{G}_{l}\left(\mathbf{q}_{l}\right) \mathbf{q}_{l}\right)\right] \\
\mathbf{M}_{r}^{-1}\left(\mathbf{q}_{r}\right)\left[\boldsymbol{\tau}_{r}-\left(\mathbf{C}_{r}\left(\mathbf{q}_{r}, \dot{\mathbf{q}}_{r}\right) \dot{\mathbf{q}}_{r}+\mathbf{G}_{r}\left(\mathbf{q}_{r}\right) \mathbf{q}_{r}\right)\right]
\end{array}\right] .
$$

The state-space equation (8) must be transformed to the SDC parameterization form (2) for left and right arm:

$$
\mathbf{A}_{i}(\mathbf{x}(t))=\left[\begin{array}{cc}
\mathbf{0}_{2 \times 2} & \mathbf{I}_{2 \times 2} \\
-\mathbf{M}_{i}^{-1}\left(\mathbf{q}_{i}\right) \mathbf{G}_{i}\left(\mathbf{q}_{i}\right) & -\mathbf{M}_{i}^{-1}\left(\mathbf{q}_{i}\right) \mathbf{C}_{i}\left(\mathbf{q}_{i}, \dot{q}_{i}\right)
\end{array}\right], \mathbf{B}_{i}(\mathbf{x}(t))=\left[\begin{array}{c}
\mathbf{0}_{2 \times 2} \\
-\mathbf{M}_{i}^{-1}\left(\mathbf{q}_{i}\right)
\end{array}\right],
$$

for $i=l, r$. The control law (4) is used for two systems

$\mathbf{u}_{i}(t)=-\mathbf{R}_{i}^{-1}(\mathbf{x}(t)) \mathbf{B}_{i}^{T}(\mathbf{x}(t)) \mathbf{K}_{i}(\mathbf{x}(t)) \mathbf{x}_{i}(t)$,

where $\mathbf{x}_{i}(t)=\left[\mathbf{q}_{i}^{T}(t), \dot{\mathbf{q}}_{i}^{T}(t)\right]^{T}$. The weighting matrix (6) is shaped as

$\mathbf{Q}_{i}(\mathbf{x}(t))=\operatorname{diag}\left(Q_{i, 11}(\mathbf{x}), Q_{i, 22}(\mathbf{x}), i, Q_{33}(\mathbf{x}), Q_{i, 44}(\mathbf{x})\right)$,

in which, based on (7), one has the penalty function as

$Q_{i, j j}(\mathbf{x}(t))=\frac{1}{\left(\sqrt{\left[x_{e, l}\left(\mathbf{q}_{l}\right)-x_{e, r}\left(\mathbf{q}_{r}\right)\right]^{2}+\left[y_{e, l}\left(\mathbf{q}_{l}\right)-y_{e, r}\left(\mathbf{q}_{r}\right)\right]^{2}}\right)^{p}}, j=1, \ldots, 4$.

The end-effector positions were set to define the distance between them as penalty functions:

$$
\begin{aligned}
& x_{e, l}\left(\mathbf{q}_{l}\right)=-d+a_{1} \cos \theta_{l, 1}+a_{2} \cos \left(\theta_{l, 1}+\theta_{l, 2}\right), \\
& x_{e, r}\left(\mathbf{q}_{r}\right)=d+a_{1} \cos \theta_{r, 1}+a_{2} \cos \left(\theta_{r, 1}+\theta_{r, 2}\right), \\
& y_{e, i}\left(\mathbf{q}_{i}\right)=a_{1} \sin \theta_{i, 1}+a_{2} \sin \left(\theta_{i, 1}+\theta_{i, 2}\right), \quad i=l, r .
\end{aligned}
$$

So, the collision avoidance term $Q_{i, j j}(\mathbf{x}(t))$, could be used in $\mathbf{Q}_{i}(\mathbf{x}(t))$ based on the desirable performance. For example, if $Q_{i, j j}(\mathbf{x}(t))$ is set on all the diagonal elements of the left arm's weighting matrix, overshoot in the response will not be observed. Or, if more maneuver of the end-effector is necessary, $Q_{i, j j}(\mathbf{x}(t))$ should be set only on the two first diagonal elements of $\mathbf{Q}_{i}(\mathbf{x}(t))$. Change in $p$ increase or decrease the speed and performance of collision avoidance. More discussion will be done in simulations.

\section{B. Hexarotors Collision-free Path Planning}

In this case, the workspace is open and free and the systems are two identical hexacopters with fixed tilted rotors. This structure provides a fully actuated structure [25]. The state vector of the system is defined as

$$
\begin{aligned}
\mathbf{x}_{i}(t) & =\left[\boldsymbol{\xi}_{i, 1}^{T}(t), \boldsymbol{\xi}_{i, 2}^{T}(t), \mathbf{v}_{i, 1}^{T}(t), \mathbf{v}_{i, 2}^{T}(t)\right]^{T} \\
& =\left[x_{i, \mathrm{c}}, y_{i, \mathrm{c}}, z_{i, \mathrm{c}}, \phi_{i}, \theta_{i}, \psi_{i}, u_{i}, v_{i}, w_{i}, p_{i}, q_{i}, r_{i}\right]^{T} .
\end{aligned}
$$

The index $i$ defines the number of the system. Considering the state vector (10) and assumption of hovering condition 
during the flights, $\dot{\xi}_{i, 2}(t) \simeq \mathbf{v}_{i, 2}(t)$ and $\dot{\xi}_{i, 1}(t) \simeq \mathbf{v}_{i, 1}(t)$ where $\dot{\xi}_{i, 2}(t)=\left[\dot{\phi}_{i}, \dot{\theta}_{i}, \dot{\psi}_{i}\right]^{T}$ and $\dot{\xi}_{i, 1}(t)=\left[\dot{x}_{i, \mathrm{c}}, \dot{y}_{i, \mathrm{c}}, \dot{z}_{i, \mathrm{c}}\right]^{T}$, the statespace representation of the system is formed [26]:

$$
\dot{\mathbf{x}}_{i}(t)=\left[\begin{array}{c}
\dot{\boldsymbol{\xi}}_{i, 1}(t) \\
\dot{\boldsymbol{\xi}}_{i, 2}(t) \\
\dot{\mathbf{v}}_{i, 1}(t) \\
\dot{\boldsymbol{v}}_{i, 2}(t)
\end{array}\right]=\left[\begin{array}{c}
\mathbf{R}_{Z Y X}\left(\boldsymbol{\xi}_{i, 2}\right) \mathbf{v}_{i, 1} \\
\mathbf{T}\left(\boldsymbol{\xi}_{i, 2}\right) \mathbf{v}_{i, 2} \\
1 / m \mathbf{I}_{3 \times 3}\left[\mathbf{R}_{Z Y X}\left(\boldsymbol{\xi}_{i, 2}\right) \mathbf{F}_{i, \mathrm{~B}}-m g \mathbf{e}_{3}-\mathbf{D} \dot{\boldsymbol{\xi}}_{i, 1}\right] \\
\mathbf{J}^{-1}\left(\boldsymbol{\xi}_{i, 2}\right)\left[\boldsymbol{\tau}_{i, \mathrm{~B}}-\mathbf{C}\left(\boldsymbol{\xi}_{i, 2}, \dot{\boldsymbol{\xi}}_{i, 2}\right) \dot{\boldsymbol{\xi}}_{i, 2}\right]
\end{array}\right],
$$

where $\mathbf{R}_{Z Y X}\left(\boldsymbol{\xi}_{i, 2}\right)$ is a rotation matrix, $m$ is the total mass of the system, $\mathbf{F}_{i, \mathrm{~B}} \in \mathbb{R}^{3}$ is input force vector, $\mathbf{D} \in \mathbb{R}^{3}$ is a diagonal matrix representing drag forces, $\mathbf{J}\left(\boldsymbol{\xi}_{i, 2}\right): \mathbb{R}^{3} \rightarrow \mathbb{R}^{3 \times 3}$ is inertia matrix of the rotational dynamics, $\boldsymbol{\tau}_{\mathrm{B}} \in \mathbb{R}^{3}$ is input torque vector, $\mathbf{C}\left(\boldsymbol{\xi}_{i, 2}, \dot{\xi}_{i, 2}\right): \mathbb{R}^{6} \rightarrow \mathbb{R}^{3 \times 3}$ is Coriolis and centrifugal forces, $\mathbf{e}_{3}=[0,0,1]^{T}$ and $\mathbf{R}_{Z Y X}\left(\boldsymbol{\xi}_{i, 2}\right)$ and $\mathbf{T}\left(\boldsymbol{\xi}_{i, 2}\right)$ could be found in Ref. [27]. The SDC parameterization of the system (11) is uniformly defined since the system is fully actuated:

$\mathbf{A}_{i}\left(\mathbf{x}_{i}\right)=\left[\begin{array}{cccc}\mathbf{0}_{3 \times 3} & \mathbf{0}_{3 \times 3} & \mathbf{R}_{Z Y X}\left(\boldsymbol{\xi}_{i, 2}\right) & \mathbf{0}_{3 \times 3} \\ \mathbf{0}_{3 \times 3} & \mathbf{0}_{3 \times 3} & \mathbf{0}_{3 \times 3} & \mathbf{T}\left(\boldsymbol{\xi}_{i, 2}\right) \\ \mathbf{0}_{3 \times 3} & \mathbf{0}_{3 \times 3} & -1 / m \mathbf{D} & \mathbf{0}_{3 \times 3} \\ \mathbf{0}_{3 \times 3} & \mathbf{0}_{3 \times 3} & \mathbf{0}_{3 \times 3} & -\mathbf{J}^{-1}\left(\boldsymbol{\xi}_{i, 2}\right) \mathbf{C}\left(\boldsymbol{\xi}_{i, 2}, \dot{\boldsymbol{\xi}}_{i, 2}\right)\end{array}\right]$,

$\mathbf{B}_{i}\left(\mathbf{x}_{i}\right)=\left[\begin{array}{cc}\mathbf{0}_{3 \times 3} & \mathbf{0}_{3 \times 3} \\ \mathbf{0}_{3 \times 3} & \mathbf{0}_{3 \times 3} \\ 1 / m \mathbf{R}_{Z X X}\left(\boldsymbol{\xi}_{i, 2}\right) & \mathbf{0}_{3 \times 3} \\ \mathbf{0}_{3 \times 3} & \mathbf{J}^{-1}\left(\boldsymbol{\xi}_{i, 2}\right)\end{array}\right]$.

The control law also excludes the gravity from the SDC matrices

$\mathbf{u}_{i}=-\left[\begin{array}{cc}\mathbf{R}_{Z Y X}^{T}\left(\mathbf{x}_{i}\right) & \mathbf{0}_{3 \times 3} \\ \mathbf{0}_{3 \times 3} & \mathbf{I}_{3 \times 3}\end{array}\right]\left(\mathbf{R}_{i}^{-1}\left(\mathbf{x}_{i}\right) \mathbf{B}_{i}^{T}\left(\mathbf{x}_{i}\right) \mathbf{K}_{i}\left(\mathbf{x}_{i}\right) \mathbf{x}_{i}+\left[\begin{array}{c}m g \mathbf{e}_{3} \\ \mathbf{0}_{3 \times 3}\end{array}\right]\right)$.

The collision scalar function is the distance between the two CoM of the UAVs

$$
Q(\mathbf{x}(t))=\frac{1}{(\underbrace{\sqrt{\left[x_{c, 1}-x_{c, 2}\right]^{2}+\left[y_{c, 1}-y_{c, 2}\right]^{2}+\left[z_{c, 1}-z_{c, 2}\right]^{2}}}_{d_{\mathrm{L}}})^{p}},
$$

with the condition $\left\{\begin{array}{lll}\text { if } & d_{\mathrm{L}}<1 & Q(\mathbf{x}(t))=\frac{1}{d_{\mathrm{L}}^{p}} \\ \text { if } & d_{\mathrm{L}}>1 & Q(\mathbf{x}(t))=d_{\mathrm{L}}^{p}\end{array}\right.$.

The scalar function then is located in the relevant diagonal arrays of the responsible UAV for collision avoidance.

\section{Simulation}

\section{A. End-effector Collision Avoidance}

The presented dynamics in Section III, a system of two manipulators are considered for simulation, with regards to the parameters in Table I. The time of simulation was set 1 second. The weighting matrices were selected as $\mathbf{R}_{r}=\mathbf{R}_{l}=0.01 \times \mathbf{I}_{2 \times 2}$ and $\quad \mathbf{Q}_{r}(\mathbf{x})=\mathbf{Q}_{l}(\mathbf{x})=10 \times \operatorname{diag}\left(L Q_{i, 11}(\mathbf{x})+1, L Q_{i, 22}(\mathbf{x})+1,1,1\right)$, where $Q_{i, 11}(\mathbf{x})=Q_{i, 22}(\mathbf{x})$ is based on (9). Start and end-point of operation were defined as $A_{l}(0,0.5) \mathrm{m}, A_{r}(0.1,0.1) \mathrm{m}$, $B_{l}(0,0) \mathrm{m}$ and $B_{r}(0,0.1) \mathrm{m}$. The trajectories of the left and right arm are presented in Fig. 2. $p$ was defined 1, and $L$ was set 1 and 0 to turn on/off the collision avoidance guard, to show that without this structure, the manipulators collided with each other in this particular example. In this example, both arms tried to protect the regulation task although the role of the left arm is more highlighted than the right due to the geometry of the start and end-point design. The distance between the endeffectors is depicted in Fig. 3.

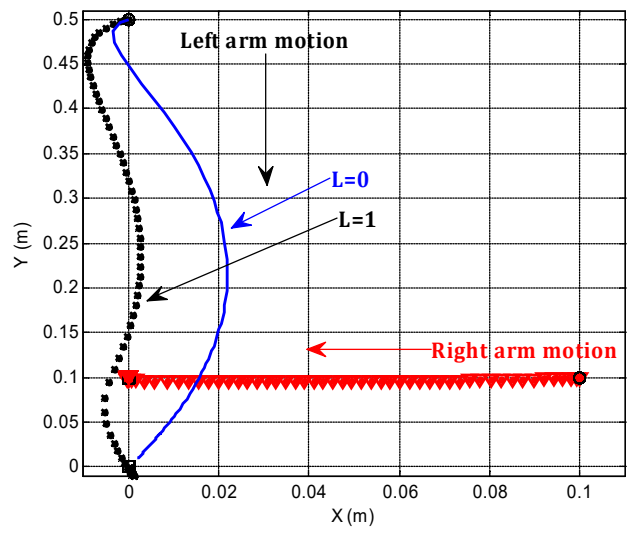

Figure 2. Simulation of collision avoidance, two planar arms.

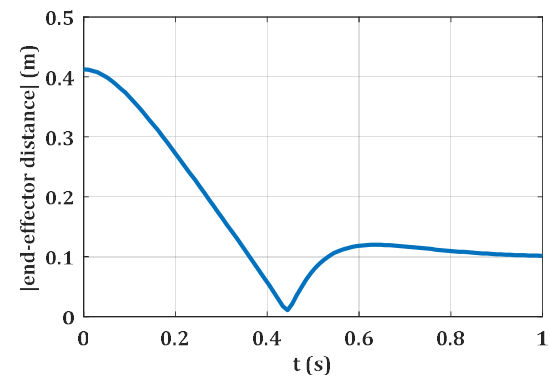

Figure 3. Distance between the end-effectors of manipulators.

The next simulation is provided to discuss the weakness of the collision avoidance of the system and emphasize on the remedy, which is the consideration of the collision avoidance term for one of the robots. The simulation was performed in 5 seconds. The weighting matrices were selected as $\mathbf{R}_{r}=\mathbf{R}_{l}=\mathbf{I}_{2 \times 2}$ and $\mathbf{Q}_{r}=\operatorname{diag}\left(Q_{i, 11}, Q_{i, 22}, Q_{i, 33}, Q_{i, 44}\right)$, where $p=5$, and:

$Q_{i, j j}(\mathbf{x}(t))=\frac{1}{\left(\sqrt{\left[x_{e, l}\left(\mathbf{q}_{l}\right)-x_{e, r}\left(\mathbf{q}_{r}\right)\right]^{2}+\left[y_{e, l}\left(\mathbf{q}_{l}\right)-y_{e, r}\left(\mathbf{q}_{r}\right)\right]^{2}}\right)^{5}}, j=1, \ldots, 4$.

The start and end-point of operation were set as $A_{l}(-0.5,0.5) \mathrm{m}, \quad A_{r}(0.5,0.5) \mathrm{m}, \quad B_{l}(0.05,0) \mathrm{m} \quad$ and $B_{r}(-0.05,0) \mathrm{m}$. In this simulation, the collision avoidance role was assigned to the right arm. The motion of the arms and the parameters are symmetric. So, they must collide without any collision avoidance structure. The trajectories were generated successfully without any collision and the right arm regulated to end-point faster than the left one to skip the impact, Fig. 4. 

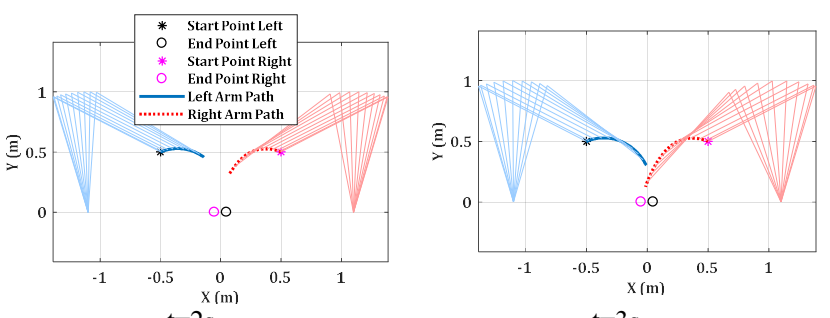

$\mathrm{t}=2 \mathrm{~s}$
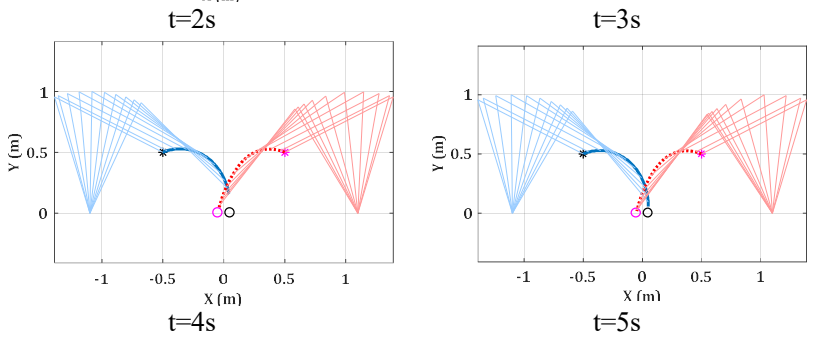

Figure 4. Symmetric regulation for verification of collision avoidance.

The verification was successfully bolded the point on one arm protection rather than two. If the safety collision terms in the structure was considered for the two arms, they would definitely collide with each other. The reason is that they both try to speed up, but the symmetric design directs them to each other.

\section{B. Hexarotors Collision-free Path Planning}

A symmetric regulation for both UAVs was designed to make sure without a collision avoidance strategy, the systems would crash. The initial and final conditions of the systems are

$\mathbf{x}_{1}(0)=\left[1, \mathbf{0}_{1 \times 1}\right]^{T}, \mathbf{x}_{2}(0)=\left[-1, \mathbf{0}_{1 \times 11}\right]^{T}$,

$\mathbf{x}_{1}(4)=\left[-1,-1.5,1.25,0.05,-0.1,0.125, \mathbf{0}_{1 \times 6}\right]^{T}$,

$\mathbf{x}_{2}(4)=\left[1,-1.5,1.25,-0.05,-0.1,0.125, \mathbf{0}_{1 \times 6}\right]^{T}$.

The mass of the system is $m=1.2(\mathrm{~kg})$, drag coefficient matrix is $\mathbf{D}=\operatorname{diag}[0.25,0.25,0.25](\mathrm{kg} / \mathrm{s})$, the distance between motor and CoM of hexacopter is $L=0.2(\mathrm{~m})$, the radius of propeller is $R=0.125(\mathrm{~m})$, inertia value about the $\mathrm{x}$-axis is $I_{x x}=7 \times 10^{-3}\left(\mathrm{kgm}^{2}\right)$, about the $\mathrm{y}$-axis is $I_{y y}=7.3 \times 10^{-3}\left(\mathrm{kgm}^{2}\right)$ and about the z-axis is $I_{y y}=3.3 \times 10^{-3}\left(\mathrm{kgm}^{2}\right)$. The weighting matrix for inputs was set $\mathbf{R}_{i}=\operatorname{diag}\left[0.01 \times \mathbf{1}_{1 \times 3}, \mathbf{1}_{1 \times 3}\right]$ for $i=1,2$. The second system is chosen for collision avoidance, hence, the weighting matrix for states are selected as

$\mathbf{Q}_{1}=\operatorname{diag}\left[\mathbf{1}_{1 \times 6}, \mathbf{0 . 5}_{1 \times 3}, \mathbf{0}_{1 \times 3}\right], \mathbf{Q}_{2}=\operatorname{diag}\left[Q \times \mathbf{1}_{1 \times 6}, \mathbf{1}_{1 \times 3},, \mathbf{0 . 5}_{1 \times 3}, \mathbf{0}_{1 \times 3}\right]$,

where $Q$ is defined based on (12) with the power of $P=7$.

The position variables of both multirotor systems are illustrated in Fig. 5. The orientation states are depicted in Fig. 6. It was possible to pose the UAVs in desired orientation due to fully actuate state of the titled systems, however, the angles must be small to satisfy the hovering condition. Configuration and trajectories of the systems are demonstrated in Fig. 7. The systems successfully regulated to the final condition without collision. The second UAV regulated faster and skipped the collision at $t=0.24 \mathrm{~s}$ when the first system was at the beginning of the motion. Distance between the systems during the control task is presented in Fig. 8 where the minimum one gained almost $0.95 \mathrm{~m}$, covering a safe distance for both drones. The obstacle avoidance term was presented in Fig. 9, showing that the function forced the second UAV to move faster at the beginning and the end of the regulation since the workspace of the operation was a large area. The minimum value was also 1 , safe for the design.

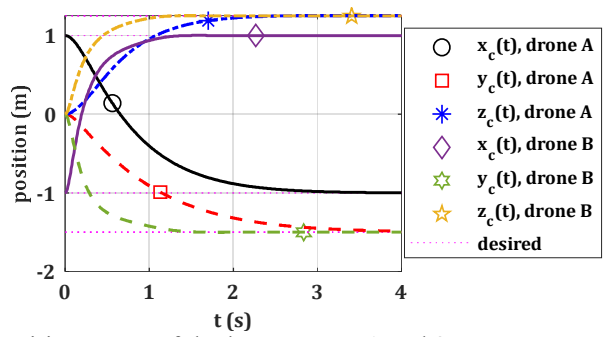

Figure 5. Position states of the hexacopters 1 and 2.

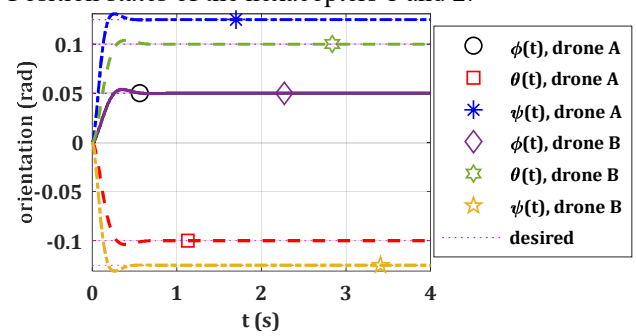

Figure 6. Orientation states of the hexacopters 1 and 2.

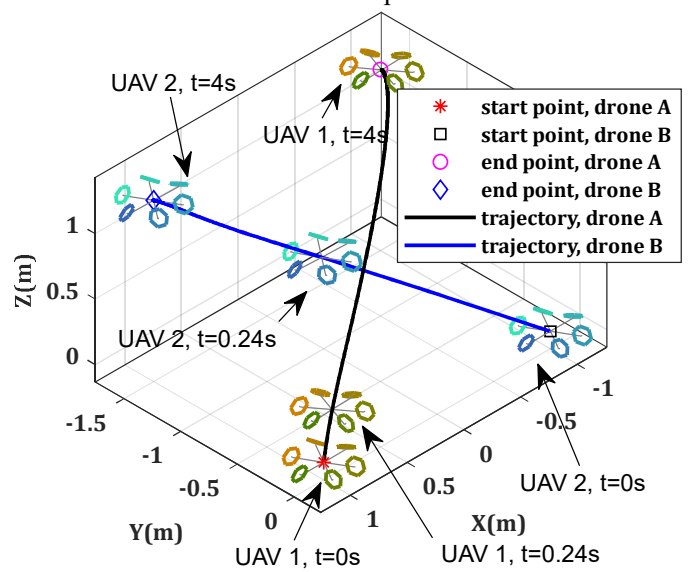

Figure 7. Configuration of UAVs and trajectories in collision avoidance.

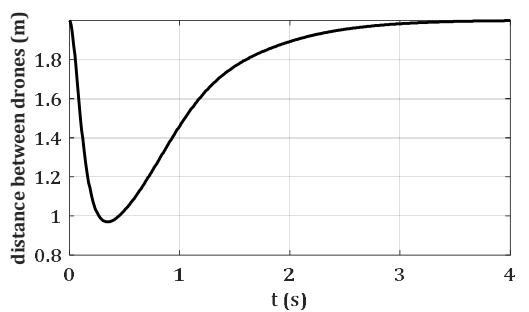

Figure 8. Distance between the UAVs.

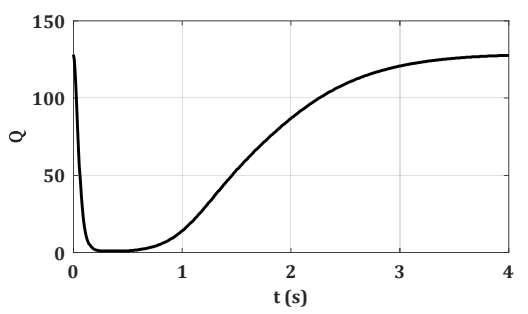

Figure 9. Obstacle avoidance scalar function. 


\section{CONCLUSIONS}

This work presented the subject of collision avoidance between the end-effectors of robotic manipulators and UAVs, working in a shared environment. The state-dependent Riccati equation was used as the control unit for the regulation problem. This controller easily accepts the artificial potential field structure in the weighting matrix of states to avoid any collision with an obstacle or possible collision with another system. The subject of obstacle avoidance was studied in the SDRE field though collision avoidance was reported once for wheeled mobile platforms. So, the end-effector collision avoidance and UAV collision avoidance were highlighted in this research. The weakness of the SDRE collision avoidance was pointed out (failure in symmetric design) and a remedy was suggested to overcome this matter (assign one system responsible for collision avoidance task). The case study was a system of two manipulators working near each other and two UAVs. Three simulations were done to show the effectiveness of the proposed design. The first one expressed a general regulation task, completed and the second a verification example to emphasize that collision avoidance function should be set on one of the arms. The symmetric design was reported as a threat to the success of the operation. The last simulation also showed collision avoidance by two UAVs in a symmetric motion. Without the collision avoidance controller, the systems would crash.

Proposal for future study: Considering the links of the manipulators in the collision avoidance function, a protecting sphere for the multirotor UAV and defining the problem for UAVs with manipulators are proposed for future research. Aerial manipulation of a system of UAVs without collision is the objective of the control approach considering safe cooperation. To increase the safety of the path planning, velocity terms could be added to the collision avoidance function to provide more design flexibility.

The proposed method was implemented for the systems of two agents. In a case that there are more than two robots, the design of a switch is necessary to assign one of the robots responsible for collision avoidance when they are close to each other. The design of the switch for the case when three agents are close is an interesting topic for future studies.

\section{ACKNOWLEDGMENT}

This work is supported by the European Commission H2020 Programme under HYFLIER project contract number 779411 and AERIAL-CORE project contract number 871479 and the Spanish project ARM-EXTEND DPI2017-89790-R.

\section{REFERENCES}

[1] S. R. Nekoo, "Tutorial and Review on the State-dependent Riccati Equation," Journal of Applied Nonlinear Dynamics, vol. 8, pp. 109-166, 2019.

[2] M. Innocenti, F. Baralli, F. Salotti, and A. Caiti, "Manipulator path control using SDRE," in Proceedings of the American Control Conference, Chicago, Illinois, 2000, pp. 3348-3352.

[3] S. Terashima, M. Iwase, K. Furuta, S. Suzuki, and S. Hatakeyama, "A design of servo controller for nonlinear systems using state dependent Riccati equation," in Proceedings of the 42nd IEEE Conference on Decision and Control, Maui, Hawaii, USA, 2003, pp. 3864-3869.

[4] A. Shawky, D. Zydek, Y. Z. Elhalwagy, and A. Ordys, "Modeling and nonlinear control of a flexible-link manipulator," Applied Mathematical Modelling, vol. 37, pp. 9591-9602, 2013.

[5] L. C. G. Souza and P. Bigot, "An adaptive method with weight matrix as a function of the state to design the rotatory flexible system control law," Mechanical Systems and Signal Processing, vol. 79, pp. 132-140, 2016.
[6] R. W. Beard and T. W. McLain, "Multiple UAV cooperative search under collision avoidance and limited range communication constraints," in $42 n d$ IEEE International Conference on Decision and Control (IEEE Cat. No. 03CH37475), Maui, Hawaii USA, 2003, pp. 25-30.

[7] X. Wang, V. Yadav, and S. N. Balakrishnan, "Cooperative UAV formation flying with obstacle/collision avoidance," IEEE Transactions on control systems technology, vol. 15, pp. 672-679, 2007.

[8] S. Pérez-Carabaza, J. Scherer, B. Rinner, J. A. López-Orozco, and E. BesadaPortas, "UAV trajectory optimization for Minimum Time Search with communication constraints and collision avoidance," Engineering Applications of Artificial Intelligence, vol. 85, pp. 357-371, 2019.

[9] K. Fathian, S. Safaoui, T. H. Summers, and N. R. Gans, "Robust 3D Distributed Formation Control With Collision Avoidance and Application to Multirotor Aerial Vehicles," in International Conference on Robotics and Automation, Montreal, Canada, 2019, pp. 9209-9215.

[10] J. Villagra, B. d'Andrea-Novel, H. Mounier, and M. Pengov, "Flatness-based vehicle steering control strategy with SDRE feedback gains tuned via a sensitivity approach," IEEE Transactions on Control Systems Technology, vol. 15 , pp. 554-565, 2007.

[11] S. R. Nekoo, "Nonlinear closed loop optimal control: A modified statedependent Riccati equation," ISA Transactions, vol. 52, pp. 285-290, 2013.

[12] M. H. Korayem, A. Zehfroosh, H. Tourajizadeh, and S. Manteghi, "Optimal motion planning of non-linear dynamic systems in the presence of obstacles and moving boundaries using SDRE: application on cable-suspended robot," Nonlinear Dynamics, vol. 76, pp. 1423-1441, 2014.

[13] Q. Ni, L. Feng, X. Chen, Y. Huang, and X. Meng, "Nonlinear optimal control of spacecraft proximity operation with obstacle constraint," in 8th International Conference on Intelligent Human-Machine Systems and Cybernetics, Zhejiang, China, 2016, pp. 266-269.

[14] S. S. Mohammadi and H. Khaloozadeh, "Optimal motion planning of unmanned ground vehicle using SDRE controller in the presence of obstacles," in 4th International Conference on Control, Instrumentation, and Automation, Qazvin, Iran, 2016, pp. 167-171.

[15] M. Alirezaei, S. T. H. Jansen, A. J. C. Schmeitz, and A. K. Madhusudhanan, "Collision avoidance system using state dependent Riccati equation technique: An experimental robustness evaluation," in Proceedings of the 13th International Symposium on Advanced Vehicle Control, Munich, Germany, 2016.

[16] Q. Ni, Y.-Y. Huang, and X.-Q. Chen, "Nonlinear control of spacecraft formation flying with disturbance rejection and collision avoidance," Chinese Physics B, vol. 26, p. 014502, 2017.

[17] M. H. Korayem and S. R. Nekoo, "The SDRE control of mobile base cooperative manipulators: Collision free path planning and moving obstacle avoidance," Robotics and Autonomous Systems, vol. 86, pp. 86-105, 2016.

[18] M. H. Korayem and S. R. Nekoo, "Nonlinear suboptimal SDRE controller for cooperative manipulators to increase dynamic load carrying capacity," in $3 r d$ RSI International Conference on Robotics and Mechatronics, Tehran, Iran, 2015, pp. 19-24.

[19] P. Huang, M. Wang, Z. Meng, F. Zhang, and Z. Liu, "Attitude takeover control for post-capture of target spacecraft using space robot," Aerospace Science and Technology, vol. 51, pp. 171-180, 2016.

[20] S. R. Nekoo, "A PDE breach to the SDRE," Asian Journal of Control, vol. 22, pp. 667-676, 2020.

[21] M. H. Korayem and S. R. Nekoo, "State-dependent differential Riccati equation to track control of time-varying systems with state and control nonlinearities," ISA Transactions, vol. 57, pp. 117-135, 2015.

[22] S. R. Nekoo and M. Irani Rahaghi, "Recursive approximate solution to timevarying matrix differential Riccati equation: linear and nonlinear systems," International Journal of Systems Science, vol. 49, pp. 2797-2807, 2018.

[23] O. Khatib, "Real-time obstacle avoidance for manipulators and mobile robots," in IEEE International Conference on Robotics and Automation., 1985, pp. 500505.

[24] M. H. Korayem and S. R. Nekoo, "Controller design of cooperative manipulators using state-dependent Riccati equation," Robotica, vol. 36, pp. 484-515, 2018.

[25] A. González Morgado, "Modelado y control de un hexarotor de hélices no coplanarias," Trabajo Fin de Grado, Dep. Ingeniería de Sistemas y Automática, Escuela Técnica Superior de Ingeniería, Universidad de Sevilla, 2018.

[26] S. R. Nekoo, J. Á. Acosta, and A. Ollero, "Fully coupled six-DoF nonlinear suboptimal control of a quadrotor: Application to variable-pitch rotor design," in Iberian Robotics conference, 2019, pp. 72-83.

[27] S. R. Nekoo, J. Á. Acosta, A. E. Gomez-Tamm, and A. Ollero, "Optimized thrust allocation of variable-pitch propellers quadrotor control: A comparative study on flip maneuver," in 2019 Workshop on Research, Education and Development of Unmanned Aerial Systems (RED UAS), Cranfield, United Kingdom, United Kingdom, 2019, pp. 86-95. 